\title{
Degenerate Saccheri Quadrilaterals, Möbius Transformations and Conjugate Möbius Transformations
}

\author{
Oğuzhan Demirel*
}

(Communicated by Yusuf Yaylı)

\begin{abstract}
In this paper, we define a new geometric concept that we will call "degenerate Saccheri quadrilateral" and use it to give a new characterization of Möbius transformations. Our proofs are based on a geometric approach.
\end{abstract}

Keywords: Saccheri quadrilateral, Möbius transformation

AMS Subject Classification (2010): Primary: 51B10 ; Secondary: 51M09; 30F45; 51M10; 51M25.

\section{Introduction}

Möbius transformations are rational functions of the form $f(z)=\frac{a z+b}{c z+d}$ satisfying $a d-b c \neq 0$, where $a, b, c, d \in$ $\mathbb{C}$. They are the automorphisms of extended complex plane $\overline{\mathbb{C}}$, that is, the meromorphic bijections $f: \overline{\mathbb{C}} \rightarrow \overline{\mathbb{C}}$. Möbius transformations are also directly conformal homeomorphisms of $\overline{\mathbb{C}}$ onto itself and they have beautiful properties. For example, a map is Möbius if, and only if it preserves cross ratios. As for geometric aspect, circlepreserving is another important characterization of Möbius transformations. There are well-known elementary proofs that if $f$ is a continuous injective map of $\overline{\mathbb{C}}$ that maps circles into circles, then $f$ is Möbius. In addition to this the following result is well known and fundamental in complex analysis.

Theorem 1.1. [1] If $f: \overline{\mathbb{C}} \rightarrow \overline{\mathbb{C}}$ is a circle preserving map, then $f$ is a Möbius transformation if and only if $f$ is a bijection.

The transformations $f(z)=\frac{a \bar{z}+b}{c \bar{z}+d}$ with $a d-b c \neq 0$, where $a, b, c, d \in \mathbb{C}$ are known as conjugate Möbius transformations of $\overline{\mathbb{C}}$. It is easy to see that each conjugate Möbius transformation $f$ is the composition of complex conjugation with a Möbius transformation, since both of these are homeomorphisms of $\overline{\mathbb{C}}$ onto itself (complex conjugation being given by reflection in the plane through $\mathbb{R} \cup\{\infty\}$ ), so is $f$. Notice that the composition of a conjugate Möbius transformation with a Möbius transformation is a conjugate Möbius transformation and composition of two conjugate Möbius transformations is a Möbius transformation. There is a topological distinction between Möbius transformations and conjugate Möbius transformations in that Möbius transformations preserve the orientation of $\overline{\mathbb{C}}$ while conjugate Möbius transformations reverse it. To see more details about conjugate Möbius transformations, we refer [11].

C. Carathéodory [4] proved that every arbitrary one to one correspondence between the points of a circular disc $C$ and a bounded point set $C^{\prime}$ by which circles lying completely in $C$ are transformed into circles lying in $C^{\prime}$ must always be either a Möbius transformation $f(z)$ or $f(\bar{z})$. R. Höfer generalized the Carathéodory's theorem to arbitrary dimensions in [9]. R. Höfer proved that for a domain $D$ of $\mathbb{R}^{n}$, if any injective mapping $f: D \rightarrow \mathbb{R}^{n}$ which takes hyperspheres whose interior is contained in $D$ to hyperspheres in $\mathbb{R}^{n}$, then $f$ is the restriction of a Möbius transformation. For more details about sphere preserving maps, see [2].

Since Möbius transformations play a major role in complex analysis and hyperbolic geometry, some authors tried to present new characterizations of Möbius transformations by using various Euclidean polygons and hyperbolic polygons. For example, in [8], H. Haruki and T.M. Rassias proved the following result by using Apollonius quadrilaterals as follows: 
Theorem 1.2. [8] Let $f: \overline{\mathbb{C}} \rightarrow \overline{\mathbb{C}}$ be a analytic and univalent transformation in a non-empty domain $R$ on the $z$-plane. Then $f$ is a Möbius transformation if and only if $f$ preserves Apollonius quadrilaterals in $R$.

S. Yang and A. Fang presented a new characterization of Möbius transformations by using Lambert quadrilaterals and Saccheri quadrilaterals in the hyperbolic plane $B^{2}=\{z:|z|<1\}$ as follows:

Theorem 1.3. [14], [15] Let $f: B^{2} \rightarrow B^{2}$ be a continuous bijection. Then $f$ is Möbius if and only if $f$ preserves Lambert quadrilaterals in $B^{2}$.

Theorem 1.4. [14], [15] Let $f: B^{2} \rightarrow B^{2}$ be a continuous bijection. Then $f$ is Möbius if and only if $f$ preserves Saccheri quadrilaterals in $B^{2}$.

Definition 1.1. [3] The Lambert quadrilateral is a hyperbolic quadrilateral with angles $\frac{\pi}{2}, \frac{\pi}{2}, \frac{\pi}{2}$ and $\theta$, where $0<\theta<\frac{\pi}{2}$.

Definition 1.2. [3] The Saccheri quadrilateral is a hyperbolic quadrilateral with angles $\frac{\pi}{2}, \frac{\pi}{2}, \theta$ and $\theta$, where $0<\theta<\frac{\pi}{2}$.

To see other characterizations of Möbius transformations with the help of hyperbolic polygons, we refer [10], [5], [6] and [7].

Distorting of the non-adjacent right angles of a Lambert quadrilateral, degenerate Lambert quadrilateral concept is defined as follows:

Definition 1.3. [7] A degenerate Lambert quadrilateral is a hyperbolic convex quadrilateral with ordered angles $\frac{\pi}{2}+\epsilon, \frac{\pi}{2}, \frac{\pi}{2}-\epsilon, \theta$ where $0<\theta<\frac{\pi}{2}$ and $0<\epsilon<\frac{\pi}{2}-\frac{\theta}{2}$.

In [7], O. Demirel proved the following result:

Theorem 1.5. [7] Let $f: B^{2} \rightarrow B^{2}$ be a surjective transformation. Then $f$ is a Möbius transformation or a conjugate Möbius transformation if and only if $f$ preserves all $\epsilon$-Lambert quadrilaterals where $0<\epsilon<\frac{\pi}{2}$.

Distorting of the right angles of a Saccheri quadrilateral, degenerate Saccheri quadrilateral concept is defined as follows:

Definition 1.4. A degenerate Saccheri quadrilateral is a hyperbolic convex quadrilateral with ordered angles $\frac{\pi}{2}-\epsilon, \frac{\pi}{2}+\epsilon, \theta, \theta$ where $0<\theta<\frac{\pi}{2}$ and $0<\epsilon<\frac{\pi}{2}-\frac{\theta}{2}$.

Notice that, for a degenerate Saccheri quadrilateral, the sum of the measures of mutual distorted angles are $\frac{\pi}{2}+\epsilon+\theta$ and $\frac{\pi}{2}-\epsilon+\theta$.

In this paper we call the degenerate Saccheri quadrilaterals having ordered angles $\frac{\pi}{2}-\epsilon, \frac{\pi}{2}+\epsilon, \theta, \theta$ briefly as $\epsilon$-Saccheri quadrilaterals. We consider the hyperbolic plane $B^{2}=\{z:|z|<1\}$ with length differential $d s^{2}=\frac{4|d z|^{2}}{\left(1-|z|^{2}\right)^{2}}$.

Throughout of the paper we denote by $X^{\prime}$ the image of $X$ under $f$, by $[P, Q]$ the geodesic segment between points $P$ and $Q$, by $P Q$ the geodesic through points $P$ and $Q$, by $P Q R$ the hyperbolic triangle with three ordered vertices $P, Q$ and $R$, by $P Q R S$ the hyperbolic quadrilateral with four ordered vertices $P, Q, R$ and $S$, and by $\angle P Q R$ the angle between $[P, Q]$ and $[P, R]$.

\section{A Characterization of Möbius Transformations by use of Degenerate Saccheri Quadrilaterals}

In this section, by the $\epsilon$-Saccheri quadrilaterals preserving property of functions, we meant that if $A B C D$ is a $\epsilon$-Saccheri quadrilateral having ordered angles $\frac{\pi}{2}-\epsilon, \frac{\pi}{2}+\epsilon, \theta$ and $\theta$, then $A^{\prime} B^{\prime} C^{\prime} D^{\prime}$ is a $\epsilon$-Saccheri quadrilateral having ordered angles $\frac{\pi}{2}-\epsilon, \frac{\pi}{2}+\epsilon, \theta^{\prime}$ and $\theta^{\prime}$.

Lemma 2.1. Let $f: B^{2} \rightarrow B^{2}$ be a mapping which preserves all $\epsilon$-Saccheri quadrilaterals where $0<\epsilon<\frac{\pi}{2}$. Then $f$ is injective.

Proof. Let us take two different points $P$ and $Q$ in $B^{2}$. Then by constructing a $\epsilon$-Saccheri quadrilateral $P Q R S$, one can easily get that $P^{\prime} Q^{\prime} R^{\prime} S^{\prime}$ is also a $\epsilon$-Saccheri quadrilateral by the property of $f$. Therefore, the points $P^{\prime}$ and $Q^{\prime}$ must be different which implies that $f$ is injective. 
Lemma 2.2. Let $f: B^{2} \rightarrow B^{2}$ be a mapping which preserves all $\epsilon$-Saccheri quadrilaterals where $0<\epsilon<\frac{\pi}{2}$. Then $f$ preserves the collinearity and betweenness properties of the points.

Proof. Let $P$ and $Q$ be two different points in $B^{2}$ and assume that $S$ is an interior point of $[P, Q]$. Then $S$ must be lie on all $\epsilon$-Saccheri quadrilaterals whose vertices are $P$ and $Q$. By the property of $f$, the images of all $\epsilon$-Saccheri quadrilaterals with vertices $P$ and $Q$ are $\epsilon$-Saccheri quadrilaterals with vertices $P^{\prime}$ and $Q^{\prime}$ and must contain $S^{\prime}$. Since $f$ is injective by Lemma 2.1, we get $P^{\prime} \neq S^{\prime} \neq Q^{\prime}$. Therefore, $S^{\prime}$ must be an interior point of $\left[P^{\prime}, Q^{\prime}\right]$ which implies that $f$ preserves the collinearity and betweenness properties of the points.

Lemma 2.3. Let $f: B^{2} \rightarrow B^{2}$ be a mapping which preserves all $\epsilon$-Saccheri quadrilaterals where $0<\epsilon<\frac{\pi}{2}$. Then $f$ preserves the angles $\frac{\pi}{2}+\epsilon$ and $\frac{\pi}{2}-\epsilon$.

Proof. Let $A B C D$ be a $\epsilon$-Saccheri quadrilateral with $\angle A B C=\frac{\pi}{2}-\epsilon, \angle B C D=\frac{\pi}{2}+\epsilon, \angle C D A=\angle D A B=\theta$ and denote the midpoint of $[B, C]$ by $M$. Now, for a fixed $\alpha \in \mathbb{R}$ satisfying $0<\alpha<\epsilon<\frac{\pi}{2}$, pick a point on $D C$, say $E$, satisfying $\angle M E C=\frac{\pi}{2}-\alpha$ and $C \in[E, D]$. Let $F$ be the common point of the geodesics $M E$ and $A B$. Notice that, if $E$ is close enough to $C$, then $F$ is close enough to $B$. Because of the fact that $\angle E C M=\angle M B F=\frac{\pi}{2}-\epsilon$, $\angle C M E=\angle B M F$ and $d_{H}=(B, M)=d_{H}(M, C)$ hold true, where $d_{H}$ is the hyperbolic distance function, by hyperbolic angle-side-angle theorem [12], the triangles $F B M$ and $E C M$ are congruent. Hence $A F E D$ must be a $\alpha$-Saccheri quadrilateral with $\angle A F E=\frac{\pi}{2}+\alpha, \angle F E D=\frac{\pi}{2}-\alpha, \angle C D A=\angle D A B=\theta$. Since $f$ preserves all degenerate Saccheri quadrilaterals, then the hyperbolic quadrilaterals $A^{\prime} B^{\prime} C^{\prime} D^{\prime}$ and $A^{\prime} F^{\prime} E^{\prime} D^{\prime}$ are $\epsilon-$ Saccheri quadrilateral and $\alpha$-Saccheri quadrilateral, respectively. Let us denote the measures of the angles of $A^{\prime} B^{\prime} C^{\prime} D^{\prime}$ and $A^{\prime} F^{\prime} E^{\prime} D^{\prime}$ by $\frac{\pi}{2}-\epsilon, \frac{\pi}{2}+\epsilon, \theta^{\prime}, \theta^{\prime}$ and $\frac{\pi}{2}-\alpha, \frac{\pi}{2}+\alpha, \theta^{\prime}, \theta^{\prime}$, respectively. Notice that since $\angle E D A=\angle C D A=$ $\angle B A D=\angle F A D=\theta$, then we have $\angle E^{\prime} D^{\prime} A^{\prime}=\angle C^{\prime} D^{\prime} A^{\prime}=\angle B^{\prime} A^{\prime} D^{\prime}=\angle F^{\prime} A^{\prime} D^{\prime}=\theta^{\prime}$. Because of the fact that $f$ preserves the collinearity and betweenness of the points by Lemma 2.2, one can easily see that the points $F^{\prime}$ and $C^{\prime}$ must be lie on $\left[A^{\prime}, B^{\prime}\right]$ and $\left[D^{\prime}, E^{\prime}\right]$, respectively. Therefore, we have $\angle A^{\prime} B^{\prime} C^{\prime}=\frac{\pi}{2}-\epsilon$ or $\angle A^{\prime} B^{\prime} C^{\prime}=\frac{\pi}{2}+\epsilon$. Now, assume that $\angle A^{\prime} B^{\prime} C^{\prime}=\frac{\pi}{2}+\epsilon$. Thus we have $\angle B^{\prime} C^{\prime} D^{\prime}=\frac{\pi}{2}-\epsilon$. Obviously, $\angle A^{\prime} F^{\prime} M^{\prime}=\frac{\pi}{2}+\alpha$ must be hold, otherwise, if $\angle A^{\prime} F^{\prime} M^{\prime}=\frac{\pi}{2}-\alpha$ holds which implies $\angle M^{\prime} F^{\prime} B^{\prime}=\frac{\pi}{2}+\alpha$, then the sum of the measures of interior angles of the triangle $F^{\prime} B^{\prime} M^{\prime}$ is greater than $\pi$ which is not possible in hyperbolic geometry. Thus we get $\angle A^{\prime} F^{\prime} M^{\prime}=\frac{\pi}{2}+\alpha$ and $\angle M^{\prime} F^{\prime} B^{\prime}=\frac{\pi}{2}-\alpha$. Let $P$ and $Q$ be the common points of the unit disc $B^{2}$ and the hyperbolic disc $D^{\prime} E^{\prime}$. Assume that $E^{\prime}$ lies on $\left[C^{\prime}, Q\right]$. If $X$ is a point moving from $Q$ to $P$ on $P Q$, then $\angle M^{\prime} X P$ must be increase from 0 to $\pi$. Hence, we get $\frac{\pi}{2}-\alpha<\frac{\pi}{2}-\epsilon$ which implies $\epsilon<\alpha$. This is a contradiction since $\alpha<\epsilon$. Therefore, we get $\angle A^{\prime} B^{\prime} C^{\prime}=\frac{\pi}{2}-\epsilon$ which implies $\angle B^{\prime} C^{\prime} D^{\prime}=\frac{\pi}{2}+\epsilon$.

Lemma 2.4. Let $f: B^{2} \rightarrow B^{2}$ be a mapping which preserves all $\epsilon$-Saccheri quadrilaterals where $0<\epsilon<\frac{\pi}{2}$. Then $f$ preserves the measures of equal angles.

Proof. Let $A B C D$ be a $\epsilon$-Saccheri quadrilateral with $\angle A B C=\frac{\pi}{2}-\epsilon, \angle B C D=\frac{\pi}{2}+\epsilon, \angle C D A=\angle D A B=\theta$. By Lemma 2.3, we have $\angle A^{\prime} B^{\prime} C^{\prime}=\frac{\pi}{2}-\epsilon, \angle B^{\prime} C^{\prime} D^{\prime}=\frac{\pi}{2}+\epsilon$. Now we have to prove that $\theta=\angle C D A=\angle D A B=$ $\angle C^{\prime} D^{\prime} A^{\prime}=\angle D^{\prime} A^{\prime} B^{\prime}=\theta^{\prime}$. Let $P Q R S$ be a hyperbolic square with center is $O$, where $O$ is the origin of $B^{2}$ satisfying $\angle P Q R=\angle Q R S=\angle R S P=\angle S P Q=\frac{\theta}{2}$. Without loss of generality, we may assume that the points $P$ and $R$ lie on the $y$-axis and the points $Q$ and $S$ lie on the $x$-axis. Let $X$ and $Y$ be the midpoints of $[P, Q]$ and $[S, R]$, respectively. Notice that the hyperbolic quadrilateral PXYS is a Saccheri quadrilateral with $\angle P X Y=\angle X Y S=\frac{\pi}{2}$ by the property of a hyperbolic square. Now, pick a point on $[S, Y]$, say $K$, such that $\angle O K Y=\theta$. The existence of $K$ is clear since $\angle O S Y=\frac{\theta}{4}$ and $\angle O Y S=\frac{\pi}{2}$. Now pick a point on $[Q, X]$, say $L$, such that $d_{H}(L, Q)=d_{H}(S, K)$. It is not hard to see that the hyperbolic triangles $O L Q$ and $O K S$ are congruent. Hence, we get $\angle O L X=\angle O K Y=\theta$. Because of $\theta<\frac{\pi}{2}$, we may represent $\theta$ as $\theta:=\frac{\pi}{2}-\alpha$. Thus we have $\angle O L Q=\pi-\theta=\pi-\left(\frac{\pi}{2}-\alpha\right)=\frac{\pi}{2}+\alpha$ which implies that $K L Q R$ is a $\alpha$-Saccheri quadrilateral with $\angle R K L=$ $\frac{\pi}{2}-\alpha, \angle K L Q=\frac{\pi}{2}+\alpha, \angle L Q R=\angle Q R K=\frac{\theta}{2}$. The angle $\angle C D A$ of the $\epsilon-$ Saccheri quadrilateral $A B C D$ can be moved to the point $K$ by an appropriate hyperbolic isometry $g$, such that the points $g(A)$ and $g(C)$ lie on the geodesics $K L$ and $K R$, respectively. Because of the fact that $f$ preserves $\epsilon$-Saccheri quadrilaterals for all $0<\epsilon<\frac{\pi}{2}$, then $K^{\prime} L^{\prime} Q^{\prime} R^{\prime}$ is a $\alpha$-Saccheri quadrilateral with $\angle L^{\prime} K^{\prime} R^{\prime}=\frac{\pi}{2}-\alpha=\theta, \angle K^{\prime} L^{\prime} Q^{\prime}=\frac{\pi}{2}+\alpha=$ $\pi-\theta, \angle L^{\prime} Q^{\prime} R^{\prime}=\angle Q^{\prime} R^{\prime} K^{\prime}$ by Lemma 2.3. Thus we get $\angle A^{\prime} D^{\prime} C^{\prime}=\angle g(A) g(D) g(C)=\angle L^{\prime} K^{\prime} R^{\prime}=\theta$ holds true. Similarly, one can easily prove that $\angle D^{\prime} A^{\prime} B^{\prime}=\theta$ holds true. Hence $f$ preserves the measures of equal angles.

Lemma 2.5. Let $f: B^{2} \rightarrow B^{2}$ be a mapping which preserves all $\epsilon$-Saccheri quadrilaterals where $0<\epsilon<\frac{\pi}{2}$. Then $f$ preserves hyperbolic distance. 
Proof. Let $A$ and $B$ be two different points in $B^{2}$. These points define two different hyperbolic squares. Choose one of them and denote it by $A B C D$. Assume $\angle A B C=\angle B C D=\angle C D A=\angle D A B:=\theta$ and denote it center by $M$. The geodesics passing through $M$, must intersect $A B C D$ at two points. Let $p$ be a hyperbolic geodesic passing through $M$ but not passing through the points $A, B, C, D, M_{1}, M_{2}, M_{3}, M_{4}$ where $M_{1}, M_{2}, M_{3}, M_{4}$ are the midpoints of $[A, B],[B, C],[C, D],[D, A]$, respectively. Therefore the hyperbolic square $A B C D$ and hyperbolic geodesic $p$ define two congruent degenerate Saccheri quadrilaterals. Assume that these degenerate Saccheri quadrilaterals be two $\beta$-Saccheri quadrilaterals. By Lemma 2.3 and Lemma 2.4, the images of these two degenerate Saccheri quadrilaterals are $\beta$-Saccheri quadrilaterals with angles $\frac{\pi}{2}-\beta, \frac{\pi}{2}+\beta, \theta, \theta$. By Lemma 2.4, we get $\angle A^{\prime} B^{\prime} C^{\prime}=\angle B^{\prime} C^{\prime} D^{\prime}=\angle C^{\prime} D^{\prime} A^{\prime}=\angle D^{\prime} A^{\prime} B^{\prime}:=\theta$. Because of the fact that the angles at the vertices of a hyperbolic square define its lengths, we get $d_{H}(A, B)=d_{H}\left(A^{\prime}, B^{\prime}\right)$, see [13].

Theorem 2.1. Let $f: B^{2} \rightarrow B^{2}$ be a surjective transformation. Then $f$ is a Möbius transformation or a conjugate Möbius transformation if and only if $f$ preserves all $\epsilon$-Saccheri quadrilaterals where $0<\epsilon<\frac{\pi}{2}$.

Proof. The "only if " part is obvious because $f$ is an isometry. Conversely, we may assume that $f$ preserves $\epsilon$-Saccheri quadrilaterals for all $0<\epsilon<\frac{\pi}{2}$ in $B^{2}$ and $f(O)=O$ by composing an hyperbolic isometry if necessary. Let us take two different points in $B^{2}$ and denote them by $x, y$. By Lemma 2.5 , we immediately get $d_{H}(O, x)=d_{H}\left(O, x^{\prime}\right)$ and $d_{H}(O, y)=d_{H}\left(O, y^{\prime}\right)$, namely $|x|=\left|x^{\prime}\right|$ and $|y|=\left|y^{\prime}\right|$, where $|\cdot|$ denotes the Euclidean norm. Therefore we get $|x-y|=\left|x^{\prime}-y^{\prime}\right|$ by since $f$ preserves angular sizes by Lemma 2.4. As

$$
2\langle x, y\rangle=|x|^{2}+|y|^{2}-|x-y|^{2}=\left|x^{\prime}\right|^{2}+\left|y^{\prime}\right|^{2}-\left|x^{\prime}-y^{\prime}\right|^{2}=2\left\langle x^{\prime}, y^{\prime}\right\rangle,
$$

$f$ preserves inner-products and then is the restriction on $B^{2}$ of an orthogonal transformation, that is, $f$ is a Möbius transformation or a conjugate Möbius transformation by Carathéodory's theorem.

Corollary 2.1. Let $f: B^{2} \rightarrow B^{2}$ be a conformal (angle preserving with sign) surjective transformation. Then $f$ is a Möbius transformation if and only if $f$ preserves all $\epsilon$-Saccheri quadrilaterals where $0<\epsilon<\frac{\pi}{2}$.

Corollary 2.2. Let $f: B^{2} \rightarrow B^{2}$ be a angle reversing surjective transformation. Then $f$ is a conjugate Möbius transformation if and only if $f$ preserves all $\epsilon$-Saccheri quadrilaterals where $0<\epsilon<\frac{\pi}{2}$.

\section{References}

[1] Aczél, J. and McKiernan, M.A., On the characterization of plane projective and complex Möbius transformations. Math. Nachr. 33, (1967), 315-337.

[2] Beardon, A.F. and Minda, D., Sphere-preserving maps in inversive geometry. Proc. Amer. Math. Soc. 130 (2002), no. 4, $987-998$.

[3] Beardon, A.F., The geometry of discrete groups, Springer-Verlag, New York, 1983.

[4] Carathéodory, C., The most general transformations of plane regions which transform circles into circles. Bull. Am. Math. Soc. 43, (1937), 573-579.

[5] Demirel, O. and Seyrantepe, E.S., A characterization of Möbius transformations by use of hyperbolic regular polygons. J. Math. Anal. Appl. 374 (2011), no. 2, 566-572.

[6] Demirel, O., A characterization of Möbius transformations by use of hyperbolic regular star polygons. Proc. Rom. Acad. Ser. A Math. Phys. Tech. Sci. Inf. Sci. 14 (2013), no. 1, 13-19.

[7] Demirel, O., Degenerate Lambert quadrilaterals and Möbius transformations. Bull. Math. Soc. Sci. Math. Roumanie, (accepted for publication).

[8] Haruki, H and Rassias, T.M., A new characteristic of Möbius transformations by use of Apollonius quadrilaterals. Proc. Amer. Math. Soc. 126 (1998), no. 10, 2857-2861.

[9] Höfer, R., A characterization of Möbius transformations. Proc. Amer. Math. Soc. 128 (2000), no. 4, 1197-1201.

[10] Jing, L., A new characteristic of Möbius transformations by use of polygons having type A. J. Math. Anal. Appl. 324 (2006), no. 1, 281-284.

[11] Jones, G.A and Singerman, D., Complex functions. An algebraic and geometric viewpoint. Cambridge University Press, Cambridge, 1987.

[12] Ungar, A.A., Analytic hyperbolic geometry. Mathematical foundations and applications. World Scientific Publishing Co. Pte. Ltd., Hackensack, NJ, 2005

[13] Ungar, A.A., The hyperbolic square and Möbius transformations, Banach J. Math. Anal. 1 (2007), no. 1, 101-116.

[14] Yang, S. and Fang, A., A new characteristic of Möbius transformations in hyperbolic geometry. J. Math. Anal. Appl. 319 (2006), no. 2, 660-664.

[15] Yang, S. and Fang, A., Corrigendum to "A new characteristic of Möbius transformations in hyperbolic geometry, J. Math. Anal. Appl. 319 (2) (2006) 660-664" J. Math. Anal. Appl. 376 (2011), no. 1, 383-384. 


\section{Affiliations}

OĞUZHAN DEMİREL

ADDRESS: Afyon Kocatepe University, Dept. of Mathematics, Afyonkarahisar-TURKEY.

E-MAIL: odemirel@aku.edu.tr

ORCID ID : orcid.org/ 0000-0001-8948-1549 